\title{
The efficacy of new oral contrast mixture for computed tomography enterography
}

\author{
Şehnaz Evrimler ${ }^{1 B, D, E, F}$, Gökhan 0 cakoğlu $^{2 C}$, 0 ktay Algin ${ }^{3 A}$ \\ 1Suleyman Demirel University, Faculty of Medicine, Department of Radiology, Isparta, Turkey \\ 'Uludag University, Faculty of Medicine, Department of Biostatistics, Bursa, Turkey \\ ${ }^{3}$ Yildirim Beyazıt University, Faculty of Medicine, Department of Radiology, Ankara, Turkey
}

\begin{abstract}
Purpose: To show the reliabilities of the new mixture (composed of water, methylcellulose, lactulose, locust bean gum, and sorbitol) and to compare the luminal distension and radiological confidence scores of this solution with water-lactulose mixture.

Material and methods: Computed tomography enterography (CTE) images in a three-year period were included randomly in our institutional review board-approved and retrospective study. Ninety-one patients drank a lactulose and water mixture (Group 1), and 54 patients drank the new mixture (Group 2). Patients who drank the oral contrast agent were taken to a 64-detector row multiple detector computed tomography machine. Coronal and sagittal reformatted images were also formed. The gastrointestinal tract was divided into 11 segments for scoring. Each segment was graded for distensional and radiological confidence. CTE images were evaluated by two radiologists.

Results: Inter- and intra-reader reliabilities were good or excellent for all gastrointestinal segments in both groups $(p<0.001)$. The best $\kappa$ values were obtained in sigmoid colon assessments. Lower agreement values were detected in duodenum and jejunum scores. The new mixture group (Group 2) showed better results than Group 1 for ileum and colonic segments according to distension and confidence scorings.
\end{abstract}

Conclusions: Inter- and intra-reader reliabilities of the new mixture were good or excellent for CTE. The new mixture seems to be more efficient and reliable for ileum and colon. The new mixture can increase bowel distention, radiological confidence, and quality in CTE evaluations.

Key words: reliability, small intestine, CT enterography, oral/rectal contrast.

\section{Introduction}

Computed tomography enterography (CTE) is an imaging technique, developed for assessing small bowel diseases. CTE is based on obtaining intravenous contrast-material enhanced abdominopelvic computed tomography (CT) images after oral contrast agent (OCA) administration for optimal small bowel distension and bowel tissue discrimination [1]. The advantages of this technique are being non-invasive, enabling high-resolution images, and the evaluation of both enteric and extra-enteric structures [2]. CTE is a more cost-effective imaging method, which provides faster imaging with higher spatial and temporal resolution than magnetic resonance elastography (MRE). Another superiority of CTE to MRE is the ability of luminal navigation. Indications are diagnosis and follow-up of inflammatory bowel diseases, diarrhoea, gastrointestinal tract bleeding, small bowel obstruction, neoplasm, polyposis syndromes, or radiation enteritis $[3,4]$. Inadequate bowel distension can result in misdiagnoses, such as; wall thickening, abnormal mural enhancement, or obscuring of intra-luminal lesions $[3,5,6]$. Polyethylenoglycol (PEG), water, methylcellulose, mannitol, and low-concentration barium with sorbitol have been studied as OCAs for CTE

\section{Correspondence address:}

Dr. Şehnaz Evrimler, Suleyman Demirel University, Faculty of Medicine, Department of Radiology, East Campus Cunur, Isparta, Turkey, e-mail: drsehnaz@gmail.com Authors' contribution:

A Study design · B Data collection · C Statistical analysis · D Data interpretation · E Manuscript preparation · F Literature search · G Funds collection 
in literature [1-4]. However, water cannot provide optimal luminal distension because of rapid absorption, and other OCAs can cause adverse effects like diarrhoea, spasm, nausea, and vomiting. Which OCAs should be used for CTE to obtain adequate luminal distension with the least adverse effect is still controversial. Therefore, we developed a new mixture of OCA, showing characteristics of both negative and positive agents, which was composed of water, methylcellulose, lactulose, locust bean gum, and sorbitol. The aim of this study was to show the characteristics of the new mixture and to compare the luminal distension and radiological confidence of the solution with the water-lactulose mixture.

\section{Material and methods}

CTE images from a three-year period (performed between 2013 and 2016) were included in our institutional review board-approved, retrospective study after informed consent was obtained. Inclusion criteria were the evaluation of diarrhoea, gastrointestinal (GI) tract bleeding, inflammatory bowel disease, coeliac disease, or small bowel tumour. Exclusion criteria were an allergy to contrast materials, renal insufficiency, pregnancy, claustrophobia, galactose intolerance, GI tract obstruction, and paediatric age. Ninety-one patients drank lactulose-water mixture (Group 1), and 54 patients drank the new mixture (Group 2). Patients were assigned to the groups in random order.

\section{Imaging protocol and preparation of the oral contrast agents}

A bowel cleansing diet and $50 \mathrm{ml}$ laxative diet solution (X-M diet solution, Yenisehir Laboratory, Ankara, Turkey) were administered to the patients the night before and on the morning of the examination. All patients were asked to drink a total of $1500 \mathrm{ml}$ of OCA in 50 minutes $300 \mathrm{ml}$ every 10 minutes. In patients with suspicious low/ moderate-grade small bowel obstruction, extra-intestinal fluid collection, fistulae, or radiation enteritis the OCA was mixed with $40 \mathrm{ml}$ Iopromide (Ultravist, Schering, Germany) in addition to the other substances, for both of the groups.

The patients in Group 1 drank $1500 \mathrm{ml}$ of OCA, composed of $200 \mathrm{ml}(670 \mathrm{mg} / \mathrm{ml})$ lactulose and $1300 \mathrm{ml}$ water. The patients in Group 2 drank a total of $1500 \mathrm{ml}$ mixture of water with $10 \mathrm{~g}$ hydroxypropyl-methylcellulose, $10 \mathrm{~g}$ lactulose, $5 \mathrm{~g}$ locust bean gum, and $5 \mathrm{~g}$ sorbitol. Although the ingredients of the mixture are well known OCAs, as far as we know they have not been used in this combination before.

Patients who drank the OCA were taken to a 64-detector row multiple detector computed tomography (MDCT) machine (Aquilion 64, Toshiba, Tokyo, Japan). Images were obtained from the level of the diaphragm to the level of the symphysis pubis, in the enteric phase (60 seconds) after the intravenous administration of $100 \mathrm{ml}$ of Iopromide (Ultravist, Schering, Germany) at a flow rate of $3 \mathrm{ml} / \mathrm{s}$. Multiphasic CTE was performed for occult GI tract bleeding. In order to decrease the intestinal motility, a spasmolytic agent, $20 \mathrm{mg}$ of hyoscine- $\mathrm{N}$-butylbromide (Buscopan, Boehringer Ingelheim Ltd, Bracknell, UK), was given intravenously immediately before the scanning. Images were obtained with $2 \mathrm{~mm}$ slice thickness and reconstructed in $1 \mathrm{~mm}$ slice thickness. Coronal and sagittal reformatted images were also formed.

\section{Image analysis}

CTE images were evaluated by two radiologists (O.A., S.E.) independently. The second observer also evaluated the same data three weeks later for the intra-observer statistics. They were blinded to the pre-diagnosis, clinical, and laboratory findings of the patients. The authors were also blinded as to which contrast agent was used. They had not seen the images before the study.

The GI tract was divided into 11 segments for scoring, as follows: stomach, duodenum, jejunum, ileum, ileocecal area, cecum, ascending colon, transverse colon, descending colon, sigmoid colon, and rectum. Each segment was graded for distension and radiological confidence. Grading of luminal distension was as follows: score 0 , collapsed segment; score 1, poor distension; score 2, moderate distension; score 3, good distension. Radiological confidence is the opinion of the observer about whether the lumen and wall of the GI segment can be evaluated with confidence in regard to the luminal distension and technical aspects. Grading of radiological confidence was scored as 0: poor, 1: good, 2: excellent.

\section{Statistical analysis}

Age values were reported as median (minimum: maximum) values and compared between groups using the MannWhitney $U$ test. Raters' scores were compared between groups by using the Pearson $\chi^{2}$ test and Freeman-Halton test. A weighted kappa ( $\kappa$ ) statistic with $95 \%$ confidence intervals was calculated for assessment of inter-reader and intra-reader reliability. Kappa coefficient less than 0.20 was interpreted as poor agreement, 0.21 to 0.40 as fair agreement, 0.41 to 0.60 as moderate agreement, 0.61 to 0.80 as good agreement, and 0.81 to 1.00 as very good agreement [7]. SPSS software version 20.0 was used for performing statistical analysis. Statistically, significance was set at $p<0.05$.

\section{Results}

The demographic characteristics of the patients were as follows; the median age of Group 1 and 2 was 53.50 (18-84) and 50.50 (22-84), respectively. Fifty-five patients were male $(61.1 \%)$ and 35 were female (38.9\%) in Group 1. Thirty-six 
of patients were male (64.8\%) and 19 were female (35.2\%) in Group 2. There was no significant difference between the groups in terms of age $(p=0.184)$ and gender $(p=0.657)$.

Inflammatory bowel disease was detected in $13 \mathrm{pa}$ tients, four of them had an enteric fistula, and one of them had a perianal fistula. GI tract tumour was detected in 12 patients. One of them was histopathologically diagnosed with FAP-associated desmoid tumour. A postoperative fistula between neobladder-ileum was depicted in a patient, whose CTE was performed for postoperative imaging.

Five patients could not drink all of the mixture because of nausea or the taste of the mixture, and two patients had diarrhoea in Group 1. Three patients could not drink all of the mixture because of nausea or the taste of the mixture, one patient vomited, and two patients had diarrhoea in Group 2. In general, the tolerability of the mixture was high. These adverse effects regressed within $24 \mathrm{~h}$.

In general, inter-reader reliabilities of distension and for radiological confidence scores were moderate or good for both groups and gastrointestinal segments $(p<0.001$, Table 1; Figures 1 and 2). Lower agreement values were detected for duodenum and jejunum scores. At the same time, the best $\kappa$ values were obtained in sigmoid colon assessments (Table 1).

According to distension scores of reader 1, Group 2 showed better results in all of the segments than Group 1, except for the duodenum, where no significant difference was observed between the groups (Table 2). The confidence scoring of the reader also showed similar results. The new mixture was more successful in all of the segments except for the duodenum and jejunum, where no significant difference was observed between the groups (Table 3, Figure 3).

Group 2 showed better results than Group 1 for ileum and colonic segments, according to the distension and confidence scorings of reader 2. Although no significant difference was found for the duodenum and jejunum, good distension and confidence agreements were observed for these segments (Table 4).

Table 1. Inter-reader reliabilities of distension and radiological confidence scores for the groups

\begin{tabular}{|c|c|c|c|c|c|c|}
\hline & \multicolumn{3}{|c|}{ Distension } & \multicolumn{3}{|c|}{ Radiological confidence } \\
\hline & \multicolumn{3}{|c|}{ Rater 1 - rater 2} & \multicolumn{3}{|c|}{ Rater 1 - rater 2} \\
\hline & к & $95 \% \mathrm{Cl}$ & $p$ & $\kappa$ & $95 \% \mathrm{Cl}$ & $p$ \\
\hline \multicolumn{7}{|l|}{ Group $1(n=91)$} \\
\hline Stomach & 0.62 & $0.48-0.76$ & $<0.001$ & 0.84 & $0.67-1.00$ & $<0.001$ \\
\hline Duodenum & 0.59 & $0.46-0.73$ & $<0.001$ & 0.68 & $0.53-0.83$ & $<0.001$ \\
\hline Jejunum & 0.52 & $0.39-0.65$ & $<0.001$ & 0.61 & $0.47-0.75$ & $<0.001$ \\
\hline Ileum & 0.47 & $0.35-0.60$ & $<0.001$ & 0.61 & $0.46-0.75$ & $<0.001$ \\
\hline Ileocecal area & 0.61 & $0.47-0.74$ & $<0.001$ & 0.53 & $0.39-0.67$ & $<0.001$ \\
\hline Cecum & 0.73 & $0.58-0.87$ & $<0.001$ & 0.75 & $0.59-0.91$ & $<0.001$ \\
\hline Ascending colon & 0.73 & $0.59-0.86$ & $<0.001$ & 0.90 & $0.75-1.00$ & $<0.001$ \\
\hline Transverse colon & 0.69 & $0.56-0.82$ & $<0.001$ & 0.90 & $0.75-1.00$ & $<0.001$ \\
\hline Descending colon & 0.75 & $0.60-0.89$ & $<0.001$ & 0.85 & $0.69-1.00$ & $<0.001$ \\
\hline Sigmoid & 0.91 & $0.76-1.00$ & $<0.001$ & 0.90 & $0.72-1.00$ & $<0.001$ \\
\hline Rectum & 0.88 & $0.73-1.00$ & $<0.001$ & 0.91 & $0.75-1.00$ & $<0.001$ \\
\hline \multicolumn{7}{|l|}{ Group $2(n=54)$} \\
\hline Stomach & 0.91 & $0.69-1.00$ & $<0.001$ & 0.60 & $0.43-0.77$ & $<0.001$ \\
\hline Duodenum & 0.44 & $0.27-0.62$ & $<0.001$ & 0.45 & $0.30-0.60$ & $<0.001$ \\
\hline Jejunum & 0.46 & $0.28-0.64$ & $<0.001$ & 0.63 & $0.43-0.82$ & $<0.001$ \\
\hline Ileum & 0.64 & $0.45-0.84$ & $<0.001$ & 0.69 & $0.45-0.83$ & $<0.001$ \\
\hline Ileocecal area & 0.22 & $0.03-0.42$ & 0.013 & 0.66 & $0.46-0.87$ & $<0.001$ \\
\hline Cecum & 0.37 & $0.17-0.56$ & $<0.001$ & 0.49 & $0.30-0.69$ & $<0.001$ \\
\hline Ascending colon & 0.46 & $0.29-0.63$ & $<0.001$ & 0.70 & $0.48-0.92$ & $<0.001$ \\
\hline Transverse colon & 0.59 & $0.42-0.76$ & $<0.001$ & 0.77 & $0.55-0.99$ & $<0.001$ \\
\hline Descending colon & 0.71 & $0.53-0.90$ & $<0.001$ & 0.89 & $0.66-1.00$ & $<0.001$ \\
\hline Sigmoid & 0.79 & $0.60-0.97$ & $<0.001$ & 0.95 & $0.72-1.00$ & $<0.001$ \\
\hline Rectum & 0.72 & $0.52-0.92$ & $<0.001$ & 0.86 & $0.63-1.00$ & $<0.001$ \\
\hline
\end{tabular}




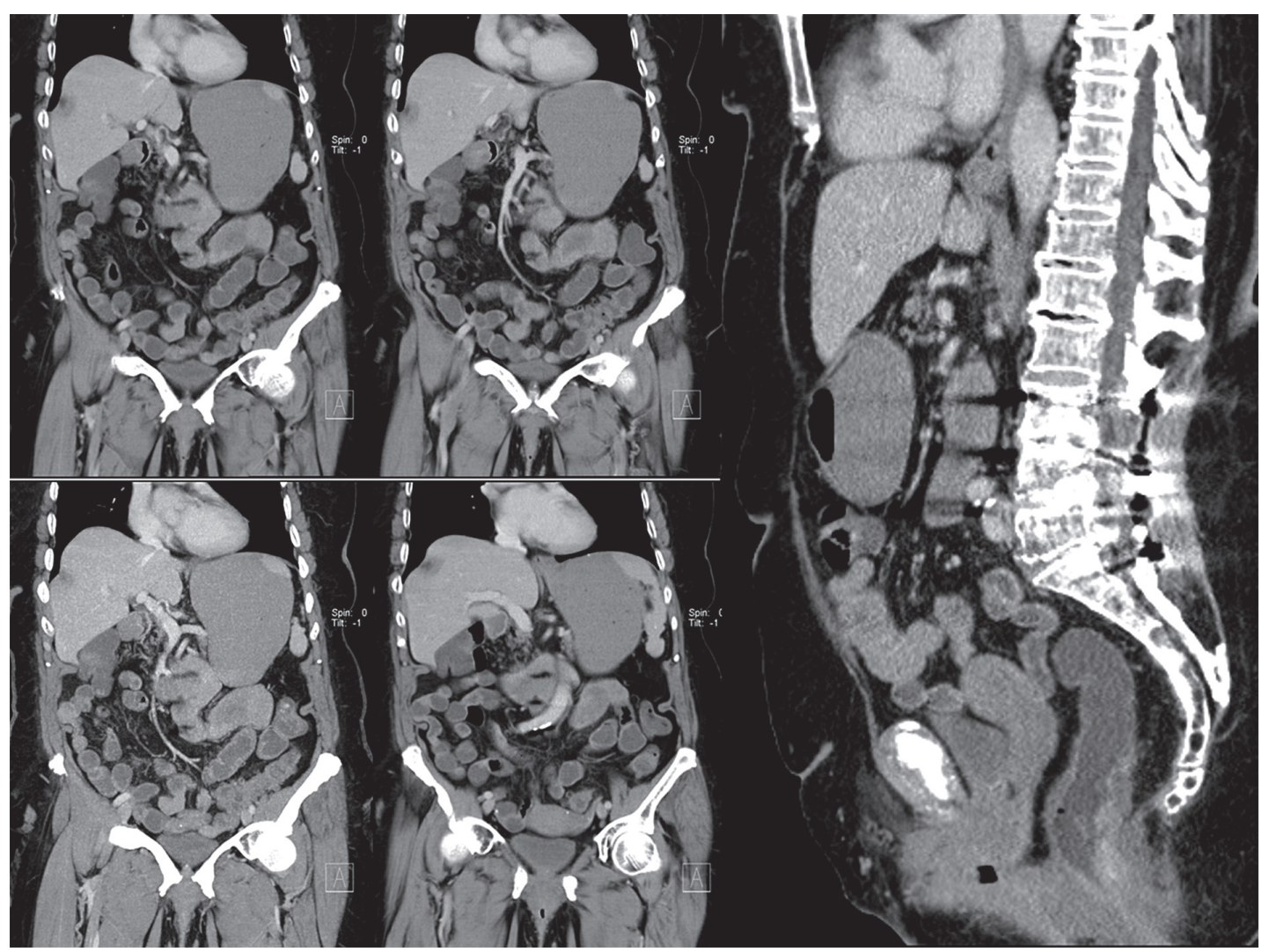

Figure 1. Computed tomography enterography (CTE), obtained with lactulose and water the new mixture, in a 66-year-old woman with elevated CEA levels and abdominal pain. In this patient, magnetic resonance elastography examination was not performed due to lumbar level metallic screws, and therefore CTE was performed. There is no abnormality in bowels or mesentery. Distension and radiological confidence scores were good to moderate for all segments

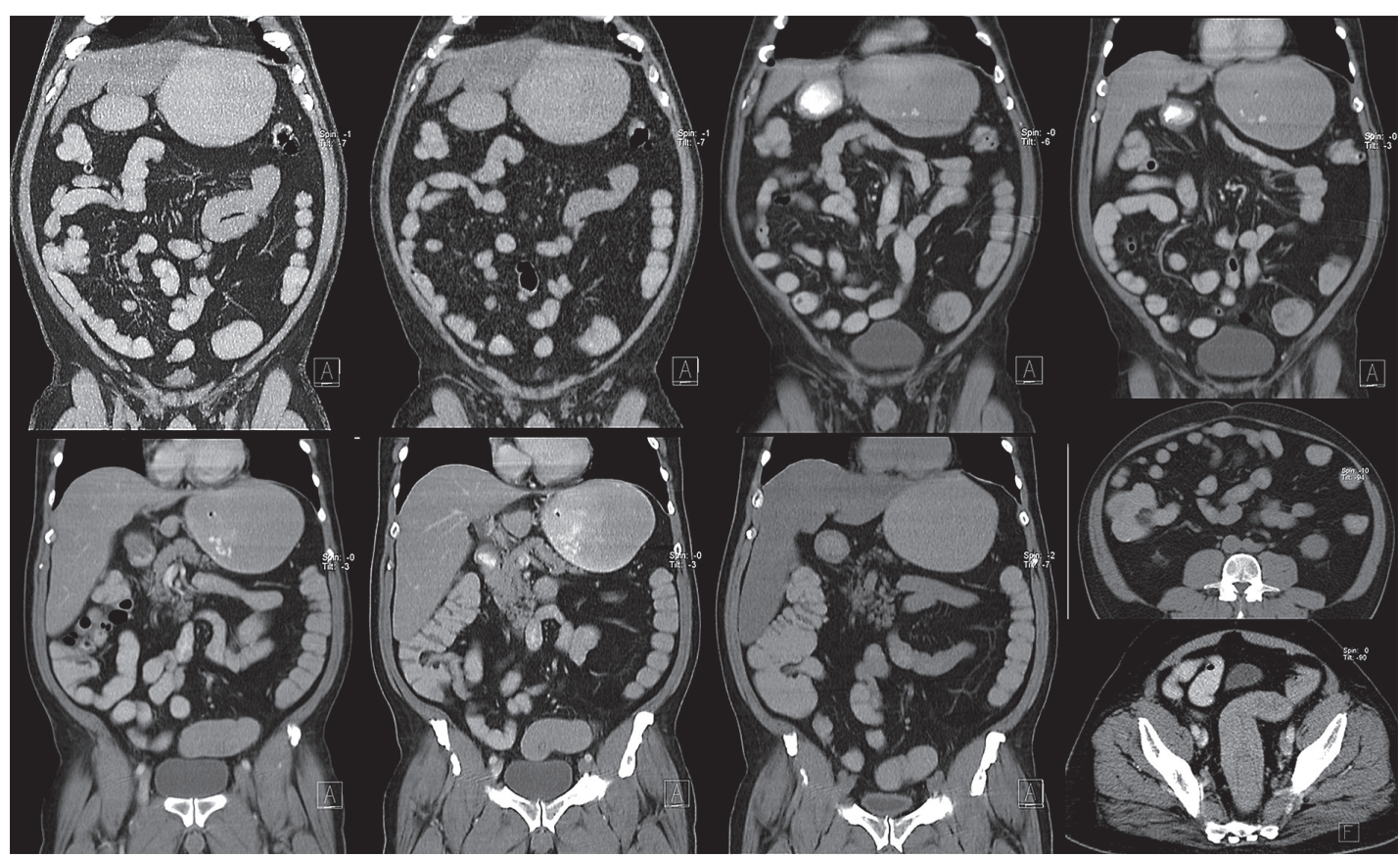

Figure 2. Computed tomography enterography, obtained with the new mixture, in a 65-year-old man with abdominal discomfort. These images reveal no abnormalities within the GIS or in the adjacent mesentery. Bowel wall thicknesses are normal, and there are good to excellent distention scores for all segments

According to the intra-reader agreement analyses of reader 2, there were excellent agreement values for each segment for all cases (Table 5). Lower agreement values were detected for duodenum and jejunum segments. At the same time, the best $\kappa$ values were obtained in sigmoid colon and rectum assessments (Table 5). 
Table 2. Distension scores of rater 1

\begin{tabular}{|c|c|c|c|c|c|c|}
\hline \multicolumn{2}{|c|}{ Distension - rater 1} & Collapsed & Moderate & Good & Excellent & $p$-value \\
\hline \multirow[t]{2}{*}{ Stomach } & Group $1(n=90)$ & $5(5.6)$ & $11(12.2)$ & 28 (31.1) & $46(51.1)$ & \multirow[t]{2}{*}{0.007} \\
\hline & Group $2(n=52)$ & $1(1.9)$ & $1(1.9)$ & $9(17.3)$ & $41(78.8)$ & \\
\hline \multirow[t]{2}{*}{ Duodenum } & Group $1(n=83)$ & $20(24.1)$ & $31(37.3)$ & $27(32.5)$ & $5(6)$ & \multirow[t]{2}{*}{0.124} \\
\hline & Group $2(n=53)$ & $8(15.1)$ & $28(52.8)$ & $11(20.8)$ & $6(11.3)$ & \\
\hline \multirow[t]{2}{*}{ Jejunum } & Group $1(n=91)$ & $16(17.6)$ & $39(42.9)$ & $27(29.7)$ & $9(9.9)$ & \multirow[t]{2}{*}{0.043} \\
\hline & Group $2(n=53)$ & $2(3.8)$ & $27(50.9)$ & $14(26.4)$ & $10(18.9)$ & \\
\hline \multirow[t]{2}{*}{ Ileum } & Group $1(n=91)$ & $9(9.9)$ & $49(53.8)$ & $24(26.4)$ & $9(9.9)$ & \multirow[t]{2}{*}{$<0.001$} \\
\hline & Group $2(n=53)$ & $0(0)$ & $14(26.4)$ & $22(41.5)$ & $17(32.1)$ & \\
\hline \multirow[t]{2}{*}{ lleocecal area } & Group $1(n=89)$ & 15 (16.9) & 37 (41.6) & $22(24.7)$ & $15(16.9)$ & \multirow[t]{2}{*}{$<0.001$} \\
\hline & Group $2(n=46)$ & $0(0)$ & $5(10.2)$ & $13(26.5)$ & $31(63.3)$ & \\
\hline \multirow[t]{2}{*}{ Cecum } & Group $1(n=90)$ & $6(6.7)$ & 55 (61.1) & $12(13.3)$ & $17(18.9)$ & \multirow[t]{2}{*}{$<0.001$} \\
\hline & Group $2(n=48)$ & $0(0)$ & $2(4.2)$ & $8(16.7)$ & $38(79.2)$ & \\
\hline \multirow[t]{2}{*}{ Ascending colon } & Group $1(n=91)$ & $11(12.1)$ & $57(62.6)$ & $8(8.8)$ & $15(16.5)$ & \multirow[t]{2}{*}{$<0.001$} \\
\hline & Group $2(n=48)$ & $2(4.2)$ & $3(6.3)$ & $11(22.9)$ & $32(66.7)$ & \\
\hline \multirow[t]{2}{*}{ Transverse colon } & Group $1(n=91)$ & $24(26.4)$ & $44(48.4)$ & $14(15.4)$ & $9(9.9)$ & \multirow[t]{2}{*}{$<0.001$} \\
\hline & Group $2(n=48)$ & $4(8.3)$ & $5(10.4)$ & $19(39.6)$ & $20(41.7)$ & \\
\hline \multirow[t]{2}{*}{ Descending colon } & Group $1(n=91)$ & $44(48.4)$ & $26(26.6)$ & $14(15.4)$ & $7(7.7)$ & \multirow[t]{2}{*}{$<0.001$} \\
\hline & Group $2(n=48)$ & $6(12.5)$ & $9(18.8)$ & $20(41.7)$ & $13(27.1)$ & \\
\hline \multirow[t]{2}{*}{ Sigmoid } & Group $1(n=85)$ & $33(38.8)$ & $31(36.5)$ & $17(20)$ & $4(4.7)$ & \multirow[t]{2}{*}{$<0.001$} \\
\hline & Group $2(n=49)$ & $6(12.2)$ & $14(28.6)$ & $14(28.6)$ & $15(30.6)$ & \\
\hline \multirow[t]{2}{*}{ Rectum } & Group $1(n=84)$ & $23(27.4)$ & $40(47.6)$ & 14 (16.7) & $7(8.3)$ & \multirow[t]{2}{*}{$<0.001$} \\
\hline & Group $2(n=48)$ & $4(8.3)$ & $10(20.8)$ & $11(22.9)$ & $23(47.9)$ & \\
\hline
\end{tabular}

Values expressed as $n(\%)$

\section{Discussion}

Our results showed that the new mixture was significantly more successful than the lactulose-water mixture according to distension and radiological confidence scores. We also observed adequate distension in colonic segments to provide optimal evaluation of colonic involvement in inflammatory diseases without the need for extra contrast agent administration. We demonstrated that the new mixture can also be used in MRE and provide adequate luminal distension [8]. Therefore, MRI can be preferred for imaging the colonic segments with late images.

There were variations in the distension scores of the intestinal segments, which might be a consequence of the variations in the intestinal motility amongst the patients. Drinking 250-500 $\mathrm{ml}$ of water in addition to the mixture just before the imaging can be useful for stomach distension or obtaining late-phase additional images can increase the colonic distension. The imaging protocol can be arranged according to the pre-diagnosis or the history of the patient.

For the most part, intra- and inter-reader reliabilities were good for the groups. Lower agreement values were detected for duodenum and jejunum scores, and the best $\kappa$ values were obtained in sigmoid colon assessments. This situation proves that the CTE technique with the new mixture is effective and reliable. The reason for the low agreement values in duodenum and jejunum may be the faster OCA discharge of these segments. If any abnormality is investigated in these regions, we think that additional fluid intake will increase the efficacy and/or reliability before the CTE.

CTE has become one of the most important imaging modalities for small bowel imaging. Endoscopy-colonoscopy, capsule endoscopy, small bowel follow-through, conventional/CT/MR enteroclysis, MRE are the other imaging techniques for evaluating small bowel diseases.

Endoscopic techniques can provide mucosal visualisation, but extra-intestinal structures cannot be evaluated. Jejunum and ileum cannot be visualised by endoscopy or colonoscopy. Also, capsule endoscopy is contraindicated for bowel obstruction and stricture suspected cases [9-11]. Enteroclysis-based techniques are centred on OCA administration via nasojejunal intubation [5]. Therefore, it is an invasive method, which provides adequate bowel distension. Also, additional ionising radiation exposure due to duodenal catheter placement is an additional disadvantage of the enteroclysis $[11,12]$. 
Table 3. Radiological confidence scores of rater 1

\begin{tabular}{|c|c|c|c|c|c|}
\hline \multicolumn{2}{|c|}{ Confidence - rater 1} & Poor & Good & Excellent & $p$-value \\
\hline \multirow[t]{2}{*}{ Stomach } & Group $1(n=90)$ & $7(7.8)$ & $18(20)$ & $65(72.2)$ & \multirow[t]{2}{*}{0.014} \\
\hline & Group $2(n=52)$ & $2(3.8)$ & $2(3.8)$ & $48(92.3)$ & \\
\hline \multirow[t]{2}{*}{ Duodenum } & Group $1(n=83)$ & $28(33.7)$ & $38(45.8)$ & $17(20.5)$ & \multirow[t]{2}{*}{0.743} \\
\hline & Group $2(n=53)$ & $21(39.6)$ & $21(39.6)$ & $11(20.8)$ & \\
\hline \multirow[t]{2}{*}{ Jejunum } & Group $1(n=91)$ & $22(24.2)$ & $44(48.4)$ & $25(27.5)$ & \multirow[t]{2}{*}{0.532} \\
\hline & Group $2(n=53)$ & $10(18.9)$ & $24(45.3)$ & $19(35.8)$ & \\
\hline \multirow[t]{2}{*}{ Ileum } & Group $1(n=91)$ & $18(19.8)$ & $48(52.7)$ & $25(27.5)$ & \multirow[t]{2}{*}{$<0.001$} \\
\hline & Group $2(n=53)$ & $1(1.9)$ & $16(30.2)$ & $36(67.9)$ & \\
\hline \multirow[t]{2}{*}{ Ileocecal area } & Group $1(n=89)$ & $22(24.7)$ & $36(40.4)$ & $31(34.8)$ & \multirow[t]{2}{*}{$<0.001$} \\
\hline & Group $2(n=49)$ & $0(0)$ & $5(10.2)$ & $44(89.8)$ & \\
\hline \multirow[t]{2}{*}{ Cecum } & Group $1(n=90)$ & $6(6.7)$ & $58(64.4)$ & $26(28.9)$ & \multirow[t]{2}{*}{$<0.001$} \\
\hline & Group $2(n=48)$ & $0(0)$ & $3(6.3)$ & $45(93.8)$ & \\
\hline \multirow[t]{2}{*}{ Ascending colon } & Group $1(n=91)$ & $14(15.4)$ & $59(64.8)$ & $18(19.8)$ & \multirow[t]{2}{*}{$<0.001$} \\
\hline & Group $2(n=48)$ & $3(6.3)$ & $6(12.5)$ & $39(81.3)$ & \\
\hline \multirow[t]{2}{*}{ Transverse colon } & Group $1(n=91)$ & $26(28.6)$ & $49(53.8)$ & $16(17.6)$ & \multirow[t]{2}{*}{$<0.001$} \\
\hline & Group $2(n=48)$ & $4(8.3)$ & $7(14.6)$ & $37(77.1)$ & \\
\hline \multirow[t]{2}{*}{ Descending colon } & Group $1(n=91)$ & $44(48.4)$ & $32(35.2)$ & $15(16.5)$ & \multirow[t]{2}{*}{$<0.001$} \\
\hline & Group $2(n=48)$ & $6(12.5)$ & $12(25)$ & $30(62.5)$ & \\
\hline \multirow[t]{2}{*}{ Sigmoid } & Group $1(n=85)$ & 35 (41.2) & $37(43.5)$ & $13(15.3)$ & \multirow[t]{2}{*}{$<0.001$} \\
\hline & Group $2(n=49)$ & $6(12.2)$ & $14(28.6)$ & $29(59.2)$ & \\
\hline \multirow[t]{2}{*}{ Rectum } & Group $1(n=84)$ & $25(29.8)$ & $46(54.8)$ & $13(15.5)$ & \multirow[t]{2}{*}{$<0.001$} \\
\hline & Group $2(n=48)$ & $4(8.3)$ & $10(20.8)$ & $34(70.8)$ & \\
\hline
\end{tabular}

Values expressed as $n(\%)$

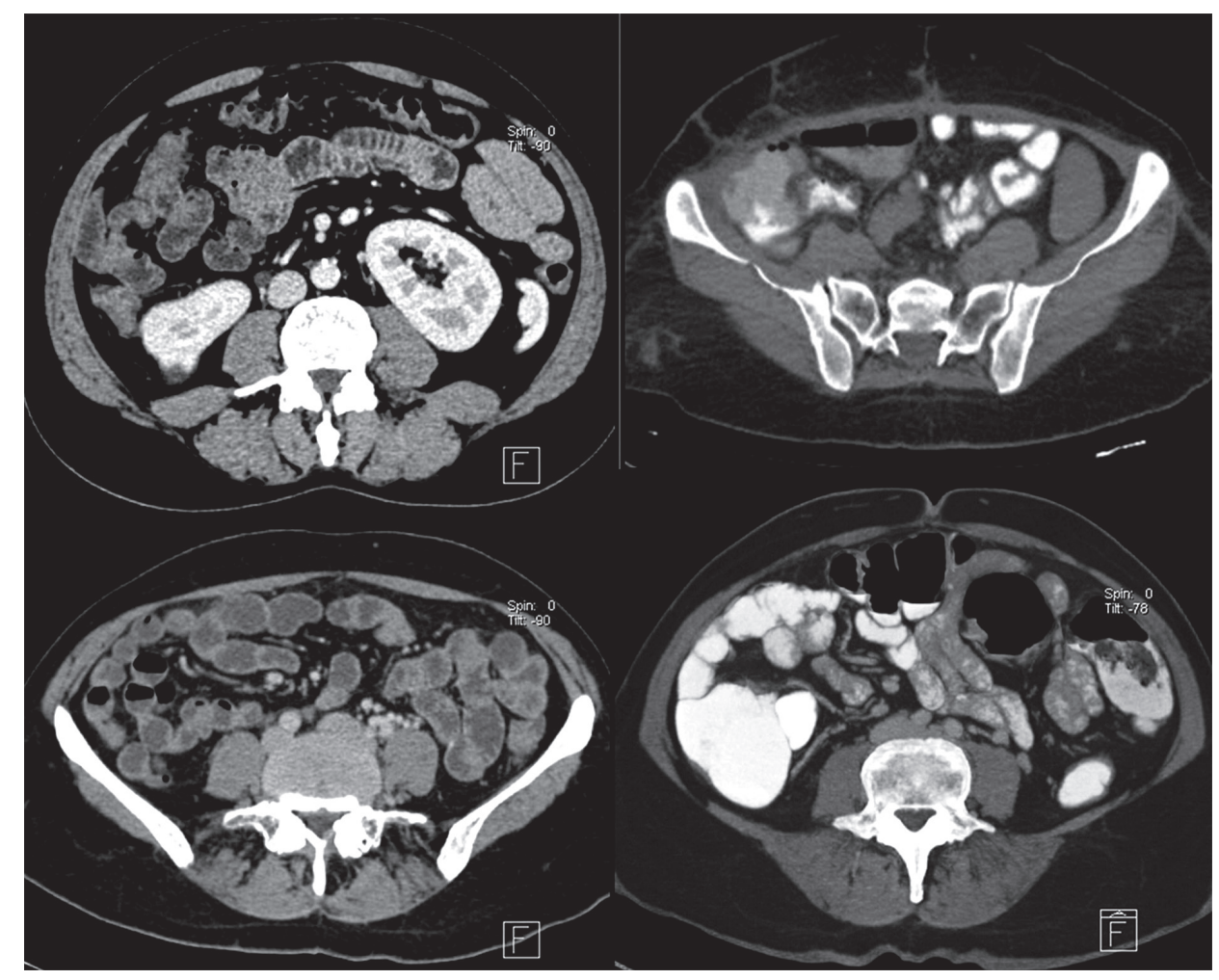

Figure 3. Computed tomography enterography images of four different patients. Upper-line images belong to Group 1 (lactulose and water mixture). Lower line images belong to Group 2 (the new mixture). The new mixture seems to be more effective in providing adequate ileal and colonic distention 
Table 4. Distension and radiological confidence scores of rater 2

\begin{tabular}{|c|c|c|c|c|c|c|}
\hline \multicolumn{2}{|c|}{ Distension - rater 2} & Collapsed & Moderate & Good & Excellent & $p$-value \\
\hline \multirow[t]{2}{*}{ Stomach } & Group $1(n=89)$ & $5(5.6)$ & $11(12.4)$ & $26(29.2)$ & $47(52.8)$ & \multirow[t]{2}{*}{0.003} \\
\hline & Group $2(n=52)$ & $1(1.9)$ & $1(1.9)$ & $7(13.5)$ & $43(87.2)$ & \\
\hline \multirow[t]{2}{*}{ Duodenum } & Group $1(n=83)$ & $5(6)$ & $35(42.2)$ & $38(45.8)$ & $5(6)$ & \multirow[t]{2}{*}{0.513} \\
\hline & Group $2(n=53)$ & $1(1.9)$ & $26(49.1)$ & $21(39.6)$ & $5(9.4)$ & \\
\hline \multirow[t]{2}{*}{ Jejunum } & Group $1(n=91)$ & $5(5.5)$ & $34(37.4)$ & $36(39.6)$ & $16(17.6)$ & \multirow[t]{2}{*}{0.403} \\
\hline & Group $2(n=53)$ & $1(1.9)$ & $15(28.3)$ & $28(52.8)$ & $9(17)$ & \\
\hline \multirow[t]{2}{*}{ Ileum } & Group $1(n=91)$ & $1(1.1)$ & $29(31.9)$ & $52(57.1)$ & $9(9.9)$ & \multirow[t]{2}{*}{0.007} \\
\hline & Group $2(n=53)$ & $0(0)$ & $7(13.2)$ & $32(60.4)$ & $14(26.4)$ & \\
\hline \multirow[t]{2}{*}{ lleocecal area } & Group $1(n=89)$ & $7(7.9)$ & $34(38.2)$ & $41(46.1)$ & $7(7.9)$ & \multirow[t]{2}{*}{$<0.001$} \\
\hline & Group $2(n=46)$ & $0(0)$ & $1(2)$ & $30(61.2)$ & $18(36.7)$ & \\
\hline \multirow[t]{2}{*}{ Cecum } & Group $1(n=90)$ & $6(6.7)$ & $41(45.6)$ & $30(33.3)$ & $13(14.4)$ & \multirow[t]{2}{*}{$<0.001$} \\
\hline & Group $2(n=48)$ & $0(0)$ & $3(6.3)$ & $23(47.9)$ & $22(45.8)$ & \\
\hline \multirow[t]{2}{*}{ Ascending colon } & Group $1(n=91)$ & $10(11)$ & $45(49.5)$ & $27(29.7)$ & $9(9.9)$ & \multirow[t]{2}{*}{$<0.001$} \\
\hline & Group $2(n=48)$ & $2(4.2)$ & $3(6.3)$ & $28(58.3)$ & $15(31.3)$ & \\
\hline \multirow[t]{2}{*}{ Transverse colon } & Group $1(n=91)$ & $18(19.8)$ & $42(46.2)$ & $25(27.5)$ & $6(6.6)$ & \multirow[t]{2}{*}{$<0.001$} \\
\hline & Group $2(n=48)$ & $4(8.3)$ & $5(10.4)$ & $30(62.5)$ & $9(18.8)$ & \\
\hline \multirow[t]{2}{*}{ Descending colon } & Group $1(n=91)$ & $34(37.4)$ & $34(37.4)$ & $20(22)$ & $3(3.3)$ & \multirow[t]{2}{*}{$<0.001$} \\
\hline & Group $2(n=48)$ & $4(8.3)$ & $10(20.8)$ & $27(56.3)$ & $7(14.6)$ & \\
\hline \multirow[t]{2}{*}{ Sigmoid } & Group $1(n=85)$ & $30(35.3)$ & $33(38.8)$ & $19(22.4)$ & $3(3.5)$ & \multirow[t]{2}{*}{$<0.001$} \\
\hline & Group $2(n=49)$ & $5(10.2)$ & $14(28.6)$ & $22(44.9)$ & $8(16.3)$ & \\
\hline \multirow[t]{2}{*}{ Rectum } & Group $1(n=84)$ & $21(25)$ & $42(50)$ & $17(20.2)$ & $4(4.8)$ & \multirow[t]{2}{*}{$<0.001$} \\
\hline & Group $2(n=48)$ & $3(6.3)$ & $11(22.9)$ & $20(41.7)$ & $14(29.2)$ & \\
\hline Confidence - rate & & Poor & Good & & & $p$-value \\
\hline Stomach & Group $1(n=89)$ & $6(6.7)$ & $26(29.2)$ & & & 0.057 \\
\hline & Group $2(n=52)$ & $1(1.9)$ & $8(15.4)$ & & & \\
\hline Duodenum & Group $1(n=83)$ & $17(20.5)$ & $51(61.4)$ & & & 0.059 \\
\hline & Group $2(n=53)$ & $3(5.7)$ & $39(73.6)$ & & & \\
\hline Jejunum & Group $1(n=91)$ & $8(8.8)$ & $60(65.9)$ & & & 0.409 \\
\hline & Group $2(n=53)$ & $2(3.8)$ & $34(64.2)$ & & & \\
\hline Ileum & Group $1(n=91)$ & $7(7.7)$ & $64(70.3)$ & & & $<0.001$ \\
\hline & Group $2(n=53)$ & $0(0)$ & $25(47.2)$ & & & \\
\hline Ileocecal area & Group $1(n=89)$ & $10(11.2)$ & $62(69.7)$ & & & $<0.001$ \\
\hline & Group $2(n=49)$ & $0(0)$ & $11(22.4)$ & & & \\
\hline Cecum & Group $1(n=90)$ & $9(10)$ & $60(66.7)$ & & & $<0.001$ \\
\hline & Group $2(n=48)$ & $0(0)$ & $9(18.8)$ & & & \\
\hline Ascending colon & Group $1(n=91)$ & $13(14.3)$ & $62(68.1)$ & & & $<0.001$ \\
\hline & Group $2(n=48)$ & $2(4.2)$ & $14(29.2)$ & & & \\
\hline Transverse colon & Group $1(n=91)$ & $23(25.3)$ & $55(60.4)$ & & & $<0.001$ \\
\hline & Group $2(n=48)$ & $4(8.3)$ & $15(31.3)$ & & & \\
\hline Descending colon & Group $1(n=91)$ & $41(45.1)$ & $40(44)$ & & & $<0.001$ \\
\hline & Group $2(n=48)$ & $5(10.4)$ & $16(33.3)$ & & & \\
\hline Sigmoid & Group $1(n=85)$ & $35(41.2)$ & $41(48.2)$ & & & $<0.001$ \\
\hline & Group $2(n=49)$ & $6(12.2)$ & $16(32.7)$ & & & \\
\hline Rectum & Group $1(n=84)$ & $23(27.4)$ & $51(60.7)$ & & & $<0.001$ \\
\hline & Group $2(n=48)$ & $3(6.3)$ & $13(27.1)$ & & & \\
\hline
\end{tabular}

Values were expressed as $n(\%)$ 
Table 5. Intra-reader reliabilities of distension and radiological confidence scores of reader 2

\begin{tabular}{|l|c|c|c|c|c|c|}
\hline \multirow{2}{*}{ Rater 2 } & \multicolumn{3}{|c|}{ Distension } & \multicolumn{3}{c|}{ Confidence } \\
\cline { 2 - 7 } & $\kappa$ & $95 \% \mathrm{Cl}$ & $\boldsymbol{p}$ & $\kappa$ & $95 \% \mathrm{Cl}$ & $p$ \\
\hline Stomach & 0.92 & $0.92-1.00$ & $<0.001$ & 0.97 & $0.83-1.00$ & $<0.001$ \\
\hline Duodenum & 0.87 & $0.75-0.99$ & $<0.001$ & 0.86 & $0.74-0.98$ & $<0.001$ \\
\hline Jejunum & 0.87 & $0.75-0.98$ & $<0.001$ & 0.85 & $0.72-0.98$ & $<0.001$ \\
\hline Ileum & 0.88 & $0.76-0.99$ & $<0.001$ & 0.87 & $0.74-1.00$ & $<0.001$ \\
\hline Ileocecal area & 0.89 & $0.77-1.00$ & $<0.001$ & 0.87 & $0.74-1.00$ & $<0.001$ \\
\hline Cecum & 0.91 & $0.79-1.00$ & $<0.001$ & 0.94 & $0.80-1.00$ & $<0.001$ \\
\hline Ascending colon & 0.88 & $0.77-1.00$ & $<0.001$ & 0.97 & $0.84-1.00$ & $<0.001$ \\
\hline Transverse colon & 0.87 & $0.76-0.98$ & $<0.001$ & 0.94 & $0.82-1.00$ & $<0.001$ \\
\hline Descending colon & 0.87 & $0.76-0.98$ & $<0.001$ & 0.96 & $0.84-1.00$ & $<0.001$ \\
\hline Sigmoid & 0.94 & $0.82-1.00$ & $<0.001$ & 0.98 & $0.85-1.00$ & $<0.001$ \\
\hline Rectum & 0.95 & $0.83-1.00$ & $<0.001$ & 0.98 & $0.85-1.00$ & $<0.001$ \\
\hline
\end{tabular}

CTE is a non-invasive, well-tolerated method that can evaluate luminal, mural, and extra-intestinal abnormalities. It provides high resolution, multiplanar reformatted images [13-15]. CTE is the fastest and the most appropriate imaging modality for evaluating active inflammatory bowel disease and its complications [16]. The only disadvantage of this technique is the radiation exposure [17]. MRE overcomes this disadvantage, so it is preferred for follow-up imaging of inflammatory bowel disease in paediatric patients $[18,19]$. On the other hand, MRE is more time consuming and expensive. The image quality is also variable in MR imaging. CTE can provide better image quality in patients who cannot hold their breath during imaging. The radiation exposure dose is the same as abdominal-pelvic venous phase CT, and it can be reduced by using a dose modulation option and advanced reconstruction techniques [20].

Inadequate luminal distension can cause misinterpretation such as wall thickening, abnormal mural enhancement, or obscuring of intraluminal lesions [5,6]. Therefore, obtaining optimal luminal distension is crucial for a good-quality CTE. OCAs used for this purpose are classified into two groups such as negative/neutral and positive. Water, lactulose, oil emulsion, Mucofalk, methylcellulose, polyethylene glycol (PEG), mannitol, locust bean gum, swollen fibre laxatives, and sorbitol are negative OCAs. Water with iodine-based contrast (ultra-low dose) agent mixtures are positive OCAs $[6,10,21,22]$.

Negative OCAs increases the visibility of mural enhancement by increasing the contrast difference between the lumen and the intestinal wall [6,9,23-28]. Positive OCAs help in the differentiation of intraluminal versus extraluminal pathologies and make the extra-intestinal fluid collections more apparent. They can obscure mural and mucosal enhancement in inflammatory bowel diseases or obscure GI tract bleeding [29,30]. Also, if the images are obtained in the arterial phase (CT angiography), these contrast agents will create difficulty in the evaluation of vascular structures [6,28,31-38]. Positive OCAs are useful for detecting the localisation of fistulas and obstruction [29].

Our new mixture is a negative OCA. If the iodine-based contrast media is mixed into the OCA, the final mixture also shows characteristics of positive OCAs, which aids in assessing extra-luminal pathologies such as fluid collections (e.g. abscess, cystic lesions, etc.), without obscuring mural or mucosal enhancement. Lactulose, sorbitol, and methylcellulose inhibit water absorption. Methylcellulose is the one with the fewest adverse effects amongst the OCAs, and hence it increases the luminal distension without increasing the adverse effects.

The optimal volume of OCA, considering adequate luminal distension, patient tolerance, and adverse effects have been discussed in the literature [39]. Maglinte et al. observed that 1.51 was not enough for small bowel distension in active inflammatory bowel disease assessment, but they also mentioned that they could have obtained good-quality images with fewer volumes [40]. Kuehle et al. used a 11 solution of $2.5 \%$ mannitol and $0.2 \%$ locust bean gum in their study and observed an increase in adverse effects but not in distension when they increased the volume to $1200-1500 \mathrm{ml}$ [41]. Boudiaf et al. performed CT enteroclysis with 21 water and observed poor distension in only two of the 107 patients in their study [24]. The amount of OCA used in studies ranges between 1300 and $2000 \mathrm{ml}[28,42-44]$, so we used $1500 \mathrm{ml}$ of OCA mixture in our study.

The limitations of the study were as follows: not all of the patients succeeded in drinking all of the solutions - some of them vomited before the imaging, but this a handicap of all of the OCAs discussed in the studies. We could only compare our mixture with water-lactulose solution because most of the other OCAs are not readily available in our country. 


\section{Conclusions}

The new mixture can provide good-quality images with optimal luminal distension. Inter- and intra-reader reliabilities of the new mixture were good or excellent (especially for ileum and colonic assessments). The new mixture is more efficient and reliable for ileum and colon. We think that using the new mixture can increase the radiological confidence in CTE evaluations.

\section{Acknowledgments}

We gratefully thank Doctor MG. Kartal and our technicians for their significant contributions.

\section{Disclosure}

The authors declare no conflicts of interest.

\section{References}

1. Evrimler S, Algin O. MR enterography with oral contrast agent composed of methylcellulose, low-dose barium sulfate, sorbitol, and lactulose: assessment of diagnostic performance, reliability, image quality, and patient tolerance. Clin Imaging 2016; 40: 523-530.

2. Algin O, Evrimler S, Ozmen E, Metin M, Ersoy O, Karaoglanoglu M. Desmoid tumor associated with familial adenomatous polyposis: evaluation with 64-detector CT enterography. Iran J Radiol 2012; 9: 32.

3. Sheedy SP, Kolbe AB, Fletcher JG, Fidler JL. Computed tomography enterography. Radiol Clin North Am 2018; 56: 649-670.

4. Algin O, Turkbey B, Ozmen E, Algin E. Magnetic resonance enterography findings of chronic radiation enteritis. Cancer Imaging 2011; 11: 189-194.

5. Masselli G, Gualdi G. Evaluation of small bowel tumors: MR enteroclysis. Abdom Imaging 2010; 35: 23-30.

6. Paulsen SR, Huprich JE, Fletcher JG, et al. CT enterography as a diagnostic tool in evaluating small bowel disorders: review of clinical experience with over 700 cases. Radiographics 2006; 26: 641-657.

7. Portney LG, Watkins MP. Foundations of Clinical Research: Applications to Practice. 2nd ed. Prentice Hall Health, Upper Saddle River 1999.

8. Algin O, Evrimler S, Ozmen E, et al. A novel biphasic oral contrast solution for enterographic studies. J Comput Assist Tomogr 2013; 37: 65-74.

9. Hara AK, Leighton JA, Heigh RI, et al. Crohn disease of the small bowel: preliminary comparison among CT enterography, capsule endoscopy, small-bowel follow-through, and ileoscopy. Radiology 2006; 238: 128-134.

10. Triester SL, Leighton JA, Leontiadis GI, et al. A meta-analysis of the yield of capsule endoscopy compared to other diagnostic modalities in patients with non-structuring small bowel Crohn's disease. Am J Gastroenterol 2006; 101: 954-964.

11. Markova I, Kluchova K, Zboril R, Mashlan M, Herman M. Small bowel imaging-still a radiologic approach. Biomed Pap Med Fac Univ Palacky Olomouc Czech Repub 2010; 154: 123-132.

12. Cronin CG, Lohan DG, Browne AM, Roche C, Murphy JM. Does MRI with oral contrast medium allow single-study depiction of inflammatory bowel disease enteritis and colitis? Eur Radiol 2010; 20: 1667-1674.

13. Hara AK, Walker FB, Silva AC, Leighton JA. Preliminary estimate of triphasic CT enterography performance in hemodynamically stable patients with suspected gastrointestinal bleeding. Am J Roentgenol 2009; 193: 1252-1260.
14. Jaffe TA, Martin LC, Miller CM, et al. Abdominal pain: coronal reformations from isotropic voxels with 16 -section CT - reader lesion detection and interpretation time. Radiology 2007; 242: 175-181.

15. Jaffe TA, Nelson RC, Johnson GA, et al. Optimization of multiplanar reformations from isotropic data sets acquired with 16-detector row helical CT scanner. Radiology 2006; 238: 292-299.

16. Kim DH, Carucci LR, Baker ME, et al. ACR Appropriateness Criteria Crohn disease. J Am Coll Radiol 2015; 12: 1048-57.e4.

17. Lee SS, Ha HK, Yang SK, et al. CT of prominent pericolic or perienteric vasculature in patients with Crohn's disease: correlation with clinical disease activity and findings on barium studies. Am J Roentgenol 2002; 179: 1029-1036.

18. Fletcher J. CT enterography technique: theme and variations. Abdom Imaging 2009; 34: 283-288.

19. Siddiki HA, Fidler JL, Fletcher JG, et al. Prospective comparison of state-of-the-art MR enterography and CT enterography in small-bowel Crohn's disease. Am J Roentgenol 2009; 193: 113-121.

20. Elsayes KM, Al-Hawary MM, Jagdish J, Ganesh HS, Platt JF. CT enterography: principles, trends, and interpretation of findings. Radiographics 2010; 30: 1955-1970.

21. Sinha R, Verma R, Verma S, Rajesh A. MR enterography of Crohn disease: part 1, rationale, technique, and pitfalls. Am J Roentgenol 2011; 197: 76-79.

22. Thomsen HS, Morcos SK, Almén T, et al. Nephrogenic systemic fibrosis and gadolinium-based contrast media: updated ESUR Contrast Medium Safety Committee guidelines. Eur Radiol 2013; 23: 307-318.

23. Arslan H, Etlik O, Kayan M, Harman M, Tuncer Yl, Temizöz O. Peroral CT enterography with lactulose solution: preliminary observations. Am J Roentgenol 2005; 185: 1173-1179.

24. Boudiaf M, Jaff A, Soyer P, Bouhnik Y, Hamzi L, Rymer R. Small-bowel diseases: prospective evaluation of multi-detector row helical CT enteroclysis in 107 consecutive patients. Radiology 2004; 233: 338-344.

25. Megibow AJ, Babb JS, Hecht EM, et al. Evaluation of bowel distention and bowel wall appearance by using neutral oral contrast agent for multi-detector row CT. Radiology 2006; 238: 87-95.

26. Raptopoulos V, Schwartz R, McNicholas M, Movson J, Pearlman J, Joffe N. Multiplanar helical CT enterography in patients with Crohn's disease. AJR Am J Roentgenol 1997; 169: 1545-1550.

27. Reittner P, Goritschnig T, Petritsch W, et al. Multiplanar spiral CT enterography in patients with Crohn's disease using a negative oral 
contrast material: initial results of a noninvasive imaging approach. Eur Radiol 2002; 12: 2253-2257.

28. Wold PB, Fletcher JG, Johnson CD, Sandborn WJ. Assessment of small bowel Crohn disease: noninvasive peroral CT enterography compared with other imaging methods and endoscopy - feasibility study. Radiology 2003; 229: 275-281.

29. Hong SS, Kim AY, Byun JH, et al. MDCT of small-bowel disease: value of 3D imaging. Am J Roentgenol 2006; 187: 1212-1221.

30. Macari M, Megibow AJ, Balthazar EJ. A pattern approach to the abnormal small bowel: observations at MDCT and CT enterography. Am J Roentgenol 2007; 188: 1344-1355.

31. Angelelli G, Macarini L. CT of the bowel: use of water to enhance depiction. Radiology 1988; 169: 848-849.

32. Bender GN, Timmons JH, Williard WC, Carter J. Computed tomographic enteroclysis: one methodology. Invest Radiol 1996; 31: 43-49.

33. Gourtsoyiannis N, Papanikolaou N, Grammatikakis J, Maris T, Prassopoulos P. MR enteroclysis protocol optimization: comparison between 3D FLASH with fat saturation after intravenous gadolinium injection and true FISP sequences. Eur Radiol 2001; 11: 908-913.

34. Horton KM, Fishman EK. The current status of multidetector row $\mathrm{CT}$ and three-dimensional imaging of the small bowel. Radiol Clin North Am 2003; 41: 199-212.

35. Lauenstein TC, Schneemann H, Vogt FM, Herborn CU, Ruhm SG, Debatin JF. Optimization of oral contrast agents for MR imaging of the small bowel. Radiology 2003; 228: 279-283.
36. Maccioni F, Viscido A, Broglia L, et al. Evaluation of Crohn disease activity with magnetic resonance imaging. Abdom Imaging 2000; 25: 219-228.

37. Mazzeo S, Caramella D, Battolla L, et al. Crohn disease of the small bowel: spiral CT evaluation after oral hyperhydration with isotonic solution. J Comput Assist Tomogr 2001; 25: 612-616.

38. Rollandi G, Curone P, Biscaldi E, et al. Spiral CT of the abdomen after distention of small bowel loops with transparent enema in patients with Crohn's disease. Abdom Imaging 1999; 24: 544-549.

39. Young B, Fletcher J, Paulsen S. Comparison of oral contrast agents for cross-sectional enterography: timing, small bowel distention and side effects. Society of Gastrointestinal Radiologists, San Antonio 2005.

40. Maglinte DD, Sandrasegaran K, Lappas JC, Chiorean M. CT enteroclysis. Radiology 2007; 245: 661-671.

41. Kuehle CA, Ajaj W, Ladd SC, Massing S, Barkhausen J, Lauenstein TC. Hydro-MRI of the small bowel: effect of contrast volume, timing of contrast administration, and data acquisition on bowel distention. Am J Roentgenol 2006; 187: W375-385.

42. Fidler JL, Guimaraes L, Einstein DM. MR imaging of the small bowel. Radiographics 2009; 29: 1811-1825.

43. Kayhan A, Oommen J, Dahi F, Oto A. Magnetic resonance enterography in Crohn's disease: standard and advanced techniques. World J Radiol 2010; 2: 113-121.

44. Schmidt S, Guibal A, Meuwly JY, et al. Acute complications of Crohn's disease: comparison of multidetector-row computed tomographic enterography with magnetic resonance enterography. Digestion 2010; 82: 229-238. 